
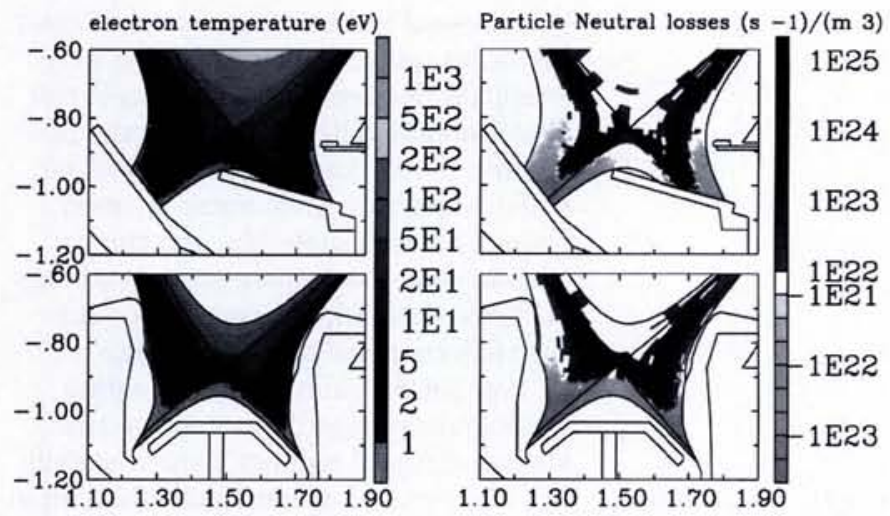

sity creates high recycling conditions: neutrals get diffusive in a random walk process, where they undergo for lower temperatures more and more $\mathrm{CX}$ collisions before they are ionized (CX larger than ionization rate coefficient). The ionization of neutrals is localized in the divertor and neutral sources get important especially for the continuity and energy equations. A recycling cycle is started, where the neutral particle sources drive ion fluxes and by thus enlarge again the neutral sources. One still gets pressure constancy along field lines, because the CX momentum losses are still negligible (a small neutral mean free path makes the divertor plasma fan opaque for neutrals). The enhanced ion divertor density gives a colder divertor with reduced physical sputtering, because the sputtering yields decrease towards lower temperature. However, this regime usually does not solve the power load problem, because the total neutral losses are limited by surface recombination of ions to a power load reduction factor of about 3 .

At rather low temperatures $(T<5 \mathrm{eV})$ the plasma detaches from the target. Momentum losses of the plasma get large by $\mathrm{CX}$ of neutrals with plasma ions and subsequent escape to the wall. A strong drop of the divertor power load is possible with the onset of strong volume recombination at temperatures of about $1 \mathrm{eV}$. The plasma recombines before reaching the target and creates a kind of virtual target. In this regime one also has very good pumping properties for helium, because recombination reduces the ion density in front of the plate and makes it easier for both the hydrogen and helium neutrals to escape out of the divertor plasma fan to the pumping ducts. The problem of this low-temperature regime is its control and stability; radiation instabilities can develop due to intrinsic impurities through a condensation instability.

Geometry strongly determines divertor effectiveness. As only a limited number of experimental configurations can be realized (money, time) validated $2 \mathrm{D}$ plasma boundary codes are used to derive optimized geometries satisfying the exhaust requirements and being compatible with core conditions.

A good example of this kind of engineering work motivated and directed by subtle physics is the change of the divertor in ASDEX Upgrade from a rather open configuration (DivI) to a strongly inclined geometry (DivII).

Comparing the contour plots of electron temperature and neutral particle sources by ionization and recombination for same upstream conditions one gets lower temperatures and a larger amount of volume recombination for DivII, especially in the high power flux region close to the separatrix. The strongly inclined target plates for DivII reflect neutrals preferentially towards this hot region and increase by this the neutral losses at the separatrix. Geometrically preferential detachment is provided and therefore a reduction of the power load at the separatrix (factor of 2 lower for DivII compared with DivI). As the outer SOL stays attached in DivII even when the separatrix zone is detached this is not changing the global detachment limit. Therefore, a maximum operational window is obtained. Additionally, better hydrogen and helium pumping is achieved.

Not yet satisfying is the need for an empirical description of the radial fluxes. Next step is to self-consistently couple turbulence codes which provide anomalous fluxes with boundary codes.

\section{Further reading}

C.S.Pitcher et al Plasma Phys. Controlled Fusion 39779 (1997)

D. Bohm The characteristics of electrical discharges in magnetic fields (Guthrie, Wakerling eds 1949)

W.- 0 . Hofer et al Physical Processes of the Interaction of Fusion Plasmas with Solids (Academic Press, London 1996)

\title{
Quasi-Symmetries in Toroidal Confinement
}

\section{Jürgen Nührenberg \\ Max-Planck-Institute für Plasma Physik, Greifswald, Germany}

Particles in toroidal plasmas with parameters typical of fusion performance have extremely long mean-free paths between collisions, typically a thousand times longer than the torus circumference. Therefore, an important aspect of toroidal plasma confinement is collision- less magnetic confinement of particle orbits. The magnetic moment of a particle, which is related to its fast gyromotion, is an adiabatic invariant and the motion of the centre of the gyro circle is called guiding-centre orbit. Together with energy conservation in a static magnetic field this invariant causes the reflection of particles which move into a region of increasing field strength for particles with sufficiently small velocities parallel to the magnetic field. This leads to the trapping of particles between regions of large field strength. In addition, guiding centre orbits experience drifts perpendicular to field lines due to field strength gradients and field line curvature. Collisions being rare, these two effects together will-in general, for arbitrary toroidal confinement regions-cause both, direct collisionless particle loss of high-energy, eg $\alpha$-particles and large diffusion coefficients of thermal particles. If the confinement domain exhibits a continuous symmetry-in a torus this is the 
Fig 1 A quasi-helically symmetric stellarator with four periods. Shown is the field strength $\mathrm{B}$ (black is low field, light grey is high field) on the plasma boundary. Note the helical structure of $B$ overriding the effect of toroidal curvature. Reflected particles drift helically

axisymmetry about the major torus axis with the toroidal angle the ignorable coordinatean additional constant of motion arises which prevents collisionless orbit loss. If the confinement region could be straight, two additional symmetries would exist: axisymmetry about the axis of the plasma column ('poloidal' symmetry) or helical symmetry. With the necessity of a toroidal confinement region (due to the intolerable end losses of a long but finite plasma column) these latter symmetries do not seem to be accessible. However, when the motion of guiding centres is described in coordinates especially adapted to the geometry of magnetic field lines ('magnetic' coordinates, in which field lines appear straight) it turns out that guiding centres do not experience the full geometrical properties of a confining magnetic field. The only three-dimensional function entering their equations of motion in these coordinates is the magnetic field strength; the flux-surface shape and other geometrical properties do not enter. So, the-at first sight counter intuitivequestion arises whether the field strength of a non-axisymmetric, ie genuinely three-dimensional, toroidal confinement domain can exhibit one of the three symmetries described above in this special representation. In essence, the answer is affirmative for the cases of axisymmetric and helically symmetric topographies of the field strength. Confinement configurations of these types are named quasi-symmetric since the symmetry does not pertain to the geometry itself. Quasi-poloidal symmetry cannot be realized but this fact led to the recognition that it would be sufficient to prevent orbit loss of a more sophisticated ersatz particle than the guiding centre particle: in connection with the trapping of particles between hills in the magnetic topography a second

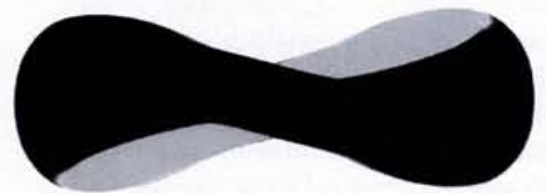

Fig 2 A quasi-axisymmetric stellarator with two periods. Even though the geometry is strongly threedimensional, reflected particles drift toroidally adiabatic invariant arises which is the action integral between reflection points; so, it suffices to have the contours of this second invariant poloidally closed. Confinement configurations of this type exist and are named quasi-isodynamic because guiding centres would only drift in magnetic surfaces (and thus behave isodynamic) if quasi-poloidal symmetry existed.

The relevance of these findings becomes apparent when considering the current state of knowledge on optimal toroidal fusion concepts: the most developed concept, the tokamak, can currently not be unequivocally identified as optimal because of its problems with disruptions (non-linear developments of instabilities leading to abrupt plasma termination) and non-inductive current drive necessary for stationary operation. On the other hand, the potential alternative avoiding these problems, the stellarator, had been plagued by its inability - which had been deemed fundamental-to confine collisionless particles without strong radial electric fields. With the results indicated above three innovative lines of stellarator research have begun to be investigated. The discovery of quasi-helically symmetric configurations (J. Nührenberg, R. Zille Phys. Lett. A 129 113, 1988) provided the first possibility of building a stellarator with collisionless particle confinement and led to the experimental realization (to be completed soon) of a small, university-scale device, the HSX (Helically Symmetric Experiment) at the University of Wisconsin, US. Figure 1 shows the topography of B at the plasma boundary; the helical feature of this quantity is obvious. The concept of quasi-axisymmetry allows the introduction into a tokamaklike (ie with significant toroidal current) configuration of externally created rotational transform (the helical twist of field lines as they go around the torus) without spoiling its particle confinement. This line, in which the danger of disruptions and the need for current drive may be mitigated, is being pursued with conceptual designs at the Princeton Plasma Physics Laboratory, US and the National Institute for Fusion Studies, Japan. Figure 2 shows the geometry and magnetic topography exhibiting the axial feature. The third confinement principle, quasiisodynamicity, is being realized in Wendelstein 7-X, a medium-sized super- conducting device designed for stationary operation being built in Greifswald. Wendelstein $7-\mathrm{X}$ exhibits the quasi-isodynamic confinement feature at finite plas$\mathrm{ma} \beta$, the ratio of plasma to magnetic field energy in the confinement domain. Figure 3 shows its geometry together with its magnetic topography exhibiting the poloidal feature for particles reflected within a period.

The complex three-dimensional features of figures 1 to 3 show that the realization of the configurational types described above required extensive computational efforts (by the standards of computing powers available in the 1980 s). They were connected with the development of the theoretical design of Wendelstein 7-X as an optimized stellarator satisfying an essential set of physics goals to be met by a stellarator viable for fusion application. These requirements pertain to equilibrium, stability and transport properties of high-temperature toroidal plasmas and it was shown that they are simultaneously satisfied in the $W_{7-X}$ configuration. Essential elements of this optimization were, on the theoretical side, qualitative knowledge about geometrical features of stellarator configurations with beneficial influence on their confinement behaviour, on the computational side, the solution of non-linear but wellposed boundary value problems for describing stellarator configurations as a basic element used in a nonlinear optimization procedure, and, on the strategic side, a risk strategy for a hierarchical attainment of physics properties, namely elimination of candidate configurations with the help of that physical property that most easily can be shown to be not attained.

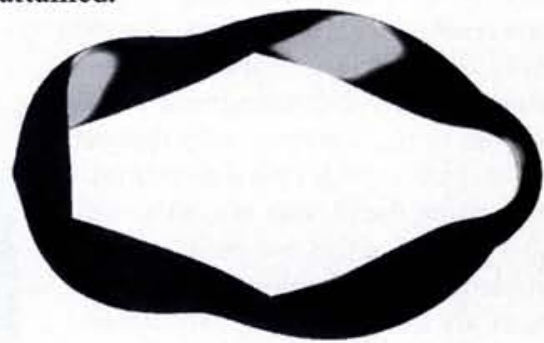

Fig 3 The Wendelstein 7-X stellarator with five periods. Particles reflected within one period drift poloidally

Further reading

J. Nührenberg, W. Lotz, S. Gori Theory of Fusion

Plasmas 3 (1994)

P.R. Garabedian Phys. Plasmas 32483 (1996)

S. Gori, W. Lotz, J. Nührenberg Theory of Fusion Plasmas 335 (1996)

W. Lotz, J, Nührenberg, C. Schwab Plasma Phys. and Contr.Nucl. Fusion Res. (Washington 1990, IAEA Vienna 1991) 2603 